\title{
Biosensor of inflammation biomarkers based on electrical bioimpedance analysis on immobilized DNA without chemical modification
}

\author{
Modesto Gómez-López ${ }^{1}$, Ángel Miliar-García ${ }^{1}$, Nadia Mabel Pérez-Vielma ${ }^{2}$, Eleazar Lara-Padilla ${ }^{1}$ \\ and César Antonio González-Díaz 1,3 \\ 1. Escuela Superior de Medicina, Instituto Politécnico Nacional. Plan de San Luis y Díaz Mirón. C.P. 11360, CDMX, México. \\ 2. Centro Interdisciplinario de Ciencias de la Salud-Unidad Santo Tomás, Instituto Politécnico Nacional. Av. Maestros C.P. 11340, CDMX, México \\ 3. E-mail any correspondence to: cgonzalezd@ipn.mx
}

\begin{abstract}
The development of biosensors to identify molecular markers or specific genes is fundamental for the implementation of new techniques that allow the detection of specific Deoxyribonucleic acid (DNA) sequences in a fast, economic and simple way. Different detection techniques have been proposed in the development of biosensors. Electrical Bioimpedance Spectroscopy (EBiS) has been used for diagnosis and monitoring of human pathologies, and is recognized as a safe, fast, reusable, easy and inexpensive technique. This study proves the development of a complementary DNA (cDNA) biosensor based on measurements of EBiS and DNA's immobilization with no chemical modifications. The evaluation of its potential utility in the detection of the gene expression of three inflammation characteristic biomarkers (NLRP3, IL-1 $\beta$ and Caspase 1 ) is presented. The obtained results demonstrate that EBiS can be used to identify different gene expression patterns, measurements that were validated by Quantitative Polymerase Chain Reaction (qPCR). These results indicate the technical feasibility for a biosensor of specific genes through bioimpedance measurements on the immobilization of CDNA.
\end{abstract}

Keywords: Biosensor; bioimpedance; gene; DNA

\section{Introduction}

Electrical Bioimpedance Spectrocopy (EBiS) also known as bioelectrical impedance analysis, has been used for diagnosis and monitoring of human pathologies and has been recognized as a safe, fast, reusable, easy and inexpensive technique. EBiS is a measurement technique based on the electrophysiological characteristics of the conductivity and dielectric properties of biological analytes $[1,2]$. Regarding the hybridization DNA sensors based on impedance, they have a wide range of applications, including medical diagnoses, biosecurity, environmental and forensics $[3,4]$. Hybridization of modified DNA with specific oligonucleotides in signaling sensors has been achieved through electrochemical impedance spectroscopy [3]. Recently, a simple electrochemical DNA-based biosensor for determining $\mathrm{Cd}^{2+}$ was reported [5], as well as an electrochemical DNA biosensor to detect gliotoxin based on DNA nanostructure-modified MXene $\left(\mathrm{Ti}_{3} \mathrm{C}_{2}\right)$ nanosheets [6]. Also, an impedimetric biosensor for the detection of DNA damage induced by doxorubicin [7].

Affinity-based biosensors may represent an alternative tool able to quantify the expression of a set of selected genes by determining the amount of corresponding messenger RNA (mRNA) in complex mixtures. The advantages of this approach include avoiding the labelling, retrotranscription, and amplification steps while maintaining high sensitivity and specificity [8-10]. Mass-sensitive biosensors have attracted considerable attention in recent years due to their simplicity, low instrumentation costs, high sensitivity, and the possibility of performing real-time and free labelling detection.

Recently, different works have been published in relation to biosensors for the detection of biomarkers related to several pathologies such as Alzheimer, cancer, and 
cardiac diseases [11-14]. Obesity is frequently associated to systemic and chronic low-grade inflammation. TNF- $\alpha$, IL-6, the inflammasome NLRP3 and the activation of proinflammatory signaling pathways have been reported in subjects and rodents with obesity [15]. The inflammasome NLRP3 is a polyprotein complex with crucial activity in the inflammatory processes [16]. This inflammasome is associated with the apoptosis-associated speck-like protein that contains a caspase recruitment domain (ASC) that recruits capase- 1 inducing its activation. Caspase- 1 is known as an IL-1 $\beta$ converting enzyme, it processes pro-IL-1 $\beta$ in its active IL-1 $\beta$ form and induces its release leading to inflammation and tissue damage $[17,18]$. These inflammation markers play an important role in several pathologies, as obesity. The traditional techniques used to determine inflammation markers are expensive, take a long time and are not reusable, which is why the use of a reliable, reusable and novel technique as EBiS might be relevant.

In this study we evaluated an innovative biosensor which development is based on two fundaments: 1) cDNA obtained from human samples, and immobilized with no chemical modification, in a functionalized gold surface and embedded in a quartz crystal; in such a way that the cDNA has the ability to hybridize with a specific oligonucleotide sequence, NLRP3, IL-1 $\beta$ and Caspase 1, those associated with inflammation, and 2) The analysis of multifrequency electrical bioimpedance changes as a function of the modification of molecular and structural components in the functionalized gold surface. Summarizing; the combination of two features are involved as fundament: 1) cDNA immobilized with no chemical modification, and 2) The analysis of multifrequency electrical bioimpedance. No complex DNA binding protocol is requiered and impedance mesurements demand minimal technical requirements, such conditions represent the innovative principle for sensing in this technological proposal to detect molecular biomarkers.

\section{Materials and methods}

\section{Description of the cDNA biosensor system}

The cDNA biosensor system consists of four modules: 1) Infusion pump, 2) Electrical-ionic interface, 3) Sciospec bioimpedance meter and 4) Personal computer (Fig. 1). The infusion pump has an insulin syringe adapted; this performs the controlled infusion of the analytes towards the electricalionic interface by means of a capillary tube. The electricalionic interface was implemented by a microfluidic module of a quartz microbalance ( $Q$ Sense-Flow, Biolin Scientific ${ }^{\mathrm{TM}}$ ). The QSense flow module is made of aluminum (shell) and titanium (liquid container), with dimesions of height; width and depth: $37 ; 35$ and $63 \mathrm{~mm}$ respectively. The volume above the electrode sensor is $40 \mu \mathrm{L}$, and the minimum sample volume is approximately $250 \mu \mathrm{L}$. The microfluidic module houses a quartz crystal disc with gold surfaces of 14 $\mathrm{mm}$ diameter as electrodes (Qfm-401, Biolin ScientificTM).
In the gold surface the cDNA immobilization and hybridization process is carried out. The flow rate was 20 $\mu \mathrm{l} / \mathrm{min}$ approximately, and it was determined on the basis of low analyte volume through the time to ensure cDNA hybridization. Thus, each step analyte infusion was performed in 12 minutes segmented by a relaxation time of 30 minutes. The Sciospec ${ }^{\mathrm{TM}}$ module (ScioSpec, ISX-3, Germany) is the instrument that allows us to interact with the electrical-ion interface module, injects a sinusoidal potential difference of $100 \mathrm{mV}$ and at the same time measures the current to estimate the impedance of the analyte. The PC is the last module, in which the programming of the ScioSpec module and storage of the data is carried out (computer is omitted in Fig. 1).

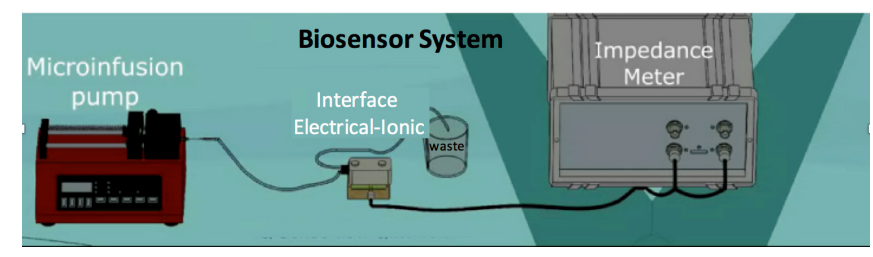

Fig. 1. Components of the cDNA biosensor system.

Patients and Blood Samples

Thirty adolescents with obesity were randomized and selected using the body mass index (BMI) criteria for classification. A blood sample $(4 \mathrm{ml})$ was obtained from the antecubital vein of the arm. RNA was extracted from the blood samples and used as template for the cDNA synthesis. A cDNA pool was made with these thirty samples, in order to determine the gene expression of NLRP3, IL-1b and Casp 1 by using the biosensor system. The gene expression determination was done by hybridizing the cDNA pool with the previously synthetized oligonucleotides specific for each of the genes. The gene expression results obtained in the biosensor system were compared with the gene expression results obtained by QPCR, a classical molecular biology technique used specifically for this purpose.

\section{Functionalization Surface Chemistry and Gold Quartz}

The reagents used for the surface chemistry over gold were 11-Mercaptoundecanoic acid (MUA), 6-Mercapto-1-hexanol (MCH), N-(3-Dimethylaminopropyl)-N'-ethylcarbodimide hydrochloride (EDC), N-Hydroxysuccinimide (NHS), and PBS 10X Buffer $\mathrm{pH}$ 7.4. All reagents for the surface chemistry were purchased from Sigma Aldrich, Mexico. All chemicals were reagent grade and used without further purification. We used quartz crystals (Q-Sense sensors) coated with 100 $\mathrm{nm}$ gold thin film, diameter $14 \mathrm{~mm}$, thickness $0.3 \mathrm{~mm}$ and optically polished, purchased from Biolin Scientific Inc., USA. The quartz crystals were washed with HPA solution and placed into UV-Ozone. After, they were incubated in a mix of MUA/MCH 1:1 solution, isolated from light for 48 hours at room temperature. Once the incubation was completed, the quartz crystals were washed with ethanol and dried with nitrogen flux. The quartz crystals were then incubated for 2 
hours, in PBS 10X (2.5 mL) with $5 \mathrm{mM}$ EDC (0.024 g) and 8 $\mathrm{mM}$ NHS $(0.023 \mathrm{~g}), \mathrm{pH} 8$. Additionally, for covalent binding of cDNA target, the crystal surfaces were exposed to $254 \mathrm{~nm}$ UV light with a power of $23 \mathrm{~mW} / \mathrm{cm}^{2}$ for 3 min by using a UV transilluminator (Stratalinker UV). The positively charged carbon atom in carbodimides rapidly reacts with the partially negatively charged oxygen in the 5'-phosphate group forming an active phosphodiester. This chemistry was previously reported by our group [19]. Diagram of functionalized gold surface quartz crystal and representation of the cDNA anchorage and as hybridization is expected is shown in Fig 2.

\section{RNA extraction and cDNA Synthesis}

Human total RNA was extracted from obese children blood samples, provided by the obesity project of the "Escuela Superior de Medicina". RNA was isolated using the TRIzol reagent according to the manufacturer's instructions (TriPure Isolation Reagent, Roche Applied Science, Indianapolis, IN). The amount and purity of RNA was quantified nanophotometrical by measuring de optical density at 260 and $280 \mathrm{~nm}$. Integrity was checked for all samples by agarose gel electrophoresis. To avoid trace amounts of DNA contamination, RNA samples were treated with amplification grade DNasel (Invitrogen, Carlsbad, CA) before reverse transcription. All RNA samples were stored at $-80^{\circ} \mathrm{C}$ in RNA elution solution until further use. First-strand cDNA Synthesis Kit (Roche Diagnostics, GmbH Mannheim, Germany) was used, taking $1 \mu \mathrm{g}$ of the total RNA sample, reverse transcription was performed with random hexamer primers for $10 \mathrm{~min}$ at $65{ }^{\circ} \mathrm{C}, 50 \mathrm{~min}$ at $35^{\circ} \mathrm{C}$ and $2 \mathrm{~min}$ at 85 ${ }^{\circ} \mathrm{C}$. DNA concentration was determined through nanospectrophometric measurement. PCRs were carried out using specific oligonucleotide primers that were generated by using the online assay design software (https://qpcr.probefinder.com/organism.jsp). After the cDNA was synthesized, a pool of the thirty patients' cDNA was made. This (350 $\mathrm{ng} / \mu \mathrm{L})$ cDNA pool was used for the bioimpedance experiments.

\section{Real-time quantitative polymerase chain reaction ( $Q P C R$ )}

PCRs were carried out using specific oligonucleotide primers that were originally generated by using the online assay design software

(https://qpcr.probefinder.com/organism.jsp).

The reaction mixture was prepared according to the manufacturer's instructions (Roche Diagnostics, $\mathrm{GmbH}$ Mannheim, Germany) using $1 \mu$ l of standard cDNA at the appropriate dilution. The amplification was performed in LightCycler 8-Tube Strips (Roche Diagnostics, $\mathrm{GmbH}$ Mannheim, Germany) with a LightCycler 2.0 instrument. Amplification conditions for the UPL-based assays were initially denaturized for $10 \mathrm{~min}$ at $95^{\circ} \mathrm{C}$ followed by 45 cycles of $10 \mathrm{~s}$ at $94^{\circ} \mathrm{C}, 20 \mathrm{~s}$ at $60^{\circ} \mathrm{C}$, and $5 \mathrm{~s}$ at $72{ }^{\circ} \mathrm{C}$. The PCR assay included standard curve of four serial dilution points for each gene, and mRNA levels were calculated by using the comparative parameter threshold cycle (Ct) method and normalized to the endogenous control, 18S rRNA. Results were calculated as a percentage of the mean level found in control using the $\Delta C T$ method.

\section{Oligonucleotides design.}

Using specific oligonucleotide primers that were originally generated by using the online assay design software (https://qpcr.probefinder.com/organism.jsp). The primer pairs used for identification the NLRP3, IL-1 $\beta$ and Caspase 1 fragment, were

5'-CACCTGTTGTGCAATCTGAAG-3' (forward) and

5'-GCAAGATCCTGACAACATGC-3' (reverse),

5'-CTGTCCTGCGTGTTGAAAGA-3' (forward) and

5'-TTGGGTAATTTTTGGGATCTAC-3' (reverse),

5'-GCGAAGCATACTTTCAGTTTC-3' (forward) and

5'-TCTCCTTCAGGACCTTGTCG-3' (reverse), respectively.

\section{Bioimpedance spectroscopy measurements}

The measurements of bioimpedance spectroscopy were made through a ScioSpec ISX3 spectrometer controlled by means of a personal PC (HP mini 110-1100), it was programmed to perform measurements in a frequency range of $100 \mathrm{~Hz}$ to $1 \mathrm{MHz}$ in 126 logarithmically spaced steps. The spectrometer injects a sinusoidal potential difference of $100 \mathrm{mV}$ and at the same time measures the current to estimate the impedance of the analyte by a two-electrode configuration. Measurements of bioimpedance were made in triplicate and stored in its resistance and reactance components, thus, EBiS magnitude estimation were determined for every gen cDNA hybridization and experimental condition.

\section{Experimental design}

The experiment to evaluate the potential utility of the cDNA biosensor system for the detection of three specific genes associated to inflammation (NLRP3, IL-1 $\beta$ and Caspase 1 ) is described as follows: The analytes were infused by a microfluidic vehicle through the electrical-ionic interface in order to perform cDNA immobilization (350 $\mathrm{ng} / \mu \mathrm{L}$ ) and hybridization with the oligonucleotide $(100 \mathrm{ng} / \mu \mathrm{L})$ in four steps: 1 ) cleaning the system with purified water (Mili-Q water), 2) cDNA immobilization on the functionalized gold surface, 3) hybridization of the CDNA with the oligonucleotide, and 4) clearance of waste with Mili-Q water and measurement of the multi-frequency electric bioimpedance on the resulting gold surface. Each step of analyte infusion was performed in 12 minutes segmented by a relaxation time of 30 minutes. In order to demonstrate changes in the electrical bioimpedance as a function of the modification of biochemical and structural components in the resulting gold surface, the experiment was designed in 
three independent tests in order to generate the following conditions: A) Control. Without immobilization of cDNA or hybridization, only Mili-Q water (vehicle) was passed in the four steps of immobilization-hybridization, so there are no structural changes in the functionalized surface. B) Genes. cDNA immobilization and hybridization, cDNA and the specific oligonucleotides were passed, so there are structural changes in the functionalized surface due to DNA complementation, and C) Negative Control. cDNA immobilization but no hybridization, cDNA and a nonspecific oligonucleotide were passed, so there are structural changes in the functionalized surface due only to the cDNA immobilization (see Fig. 2). Each assay was performed in triplicate. When obtaining the measurements of both the resistive and the reactive parts, the Nyquiste plot of each one was determined, after this an average of the three measurements was made.

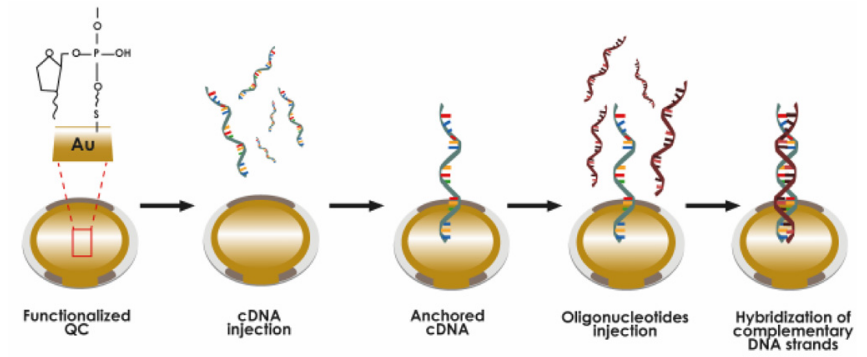

Fig. 2. Diagram of functionalized gold surface quartz crystal and representation of the CDNA anchorage and hybridization.

\section{Informed consent}

Written and informed consent was obtained from all participants.

\section{Ethical approval}

This protocol was approved by the ethics and research committees of the "Escuela Superior de Medicina" (ESMCE-01/03-12-2013). The trial was conducted in accordance with the ethical principles originated in the Declaration of Helsinki, and it was consistent with Good Clinical Practice Guidelines.

\section{Results}

Fig. 3 shows the bioimpedance spectra in the Nyquiste plot. The control test shows a substantially different behaviour with respect to the genes and negative control assays. We observed that for the genes assay and the negative control there is a notable difference in the results due to the existence of hybridization and the absence of it for the negative control case, the inflexion points represent zero phase as maximum value.

\section{EBiS vs $q P C R$}

As strategy to quantify EBiS in one simple value, EBiS spectra magnitude measurements were integrated in as area under the curve $\left(A_{z}\right)$ in the frequency $(f)$ range of $100 \mathrm{~Hz}-1 \mathrm{MHz}$, according to the following equation:

$$
\boldsymbol{A}_{Z}=\int_{0.0001}^{1} Z(f) d f
$$

Then a relative ratio with respect the observed measurements for the non-specific gen was calculated and compared with the expression of mRNA (Fig. 4). Integrated relative EBiS shows differential values for every gene in agreement with the expression of the genes encoding for NLRP3, IL-1 $\beta$, and Casp-1. An ANOVA test indicates statistical differences between groups for integrated relative $\mathrm{EBiS}$ and relative mRNA expression with $p<0.01$ and $p<0.05$, respectively.

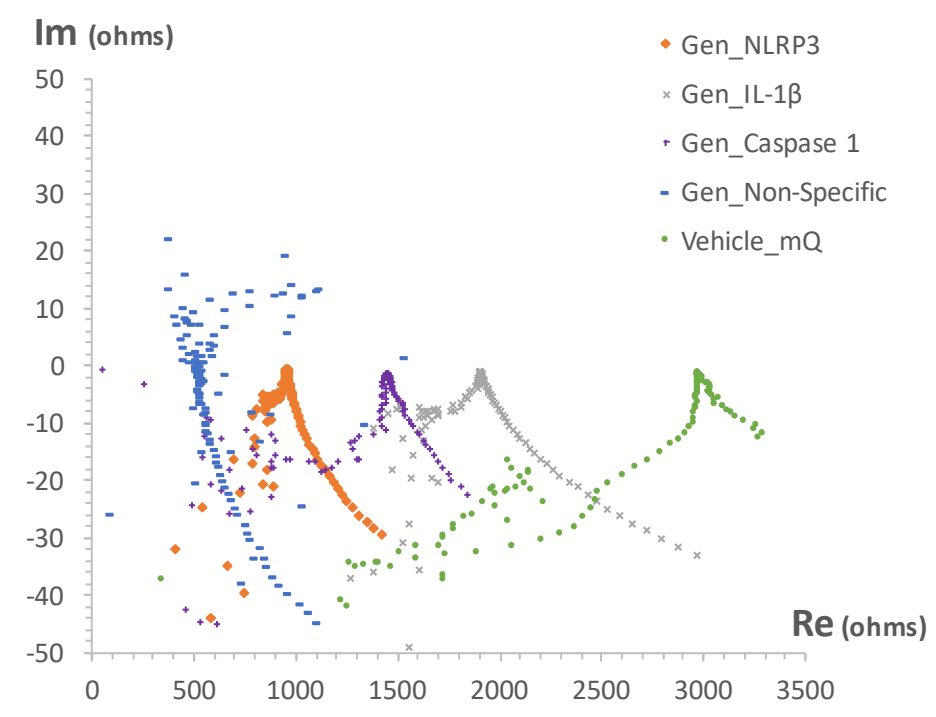

Fig. 3. Nyquiste plot EBiS spectra for the three genes studied (NLRP3, IL-1B and Caspase 1) as well as control (vehicle) and negative control (non-specific gene) conditions.

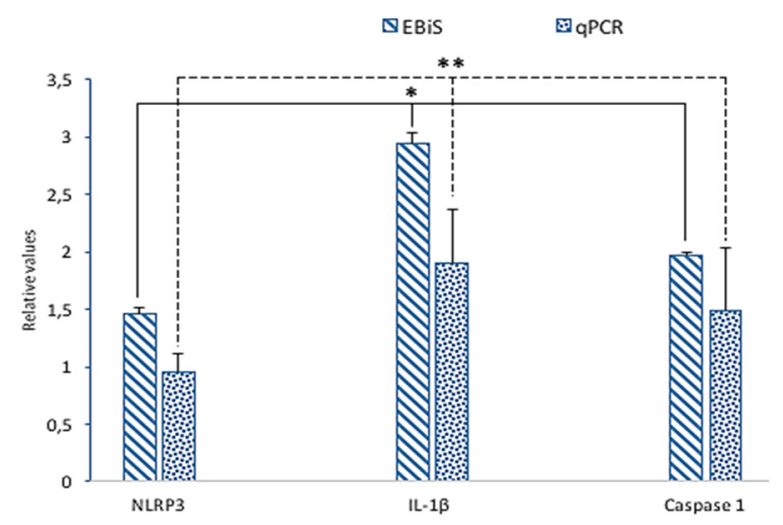

Fig. 4. Relative integrated EBiS vs relative $q P C R$ expression of the genes NLRP3, IL-16, and Casp-1. One-way ANOVA test ${ }^{*} p<0.01,{ }^{*} p<0.05$.

\section{Discussion}

The search for biomarkers to support the diagnosis and understanding of the pathophysiology of human diseases is becoming increasingly important in the medical area. The development of different types of biosensors in this area has increased in the past years due to the advantages offered by 
biosensors; low quantity sample, onsite detection, quick and robust analysis [14]. EBis is recognized as a safe, fast, reusable, easy, inexpensive and simple to perform technique, requiring no detection tags, also allowing for miniaturization $[14,20]$. Due to these advantages, EBiS is the technique preferred for label-free detections and for biorecognitions events such as protein immobilization, antibody affinity, enzyme activity, DNA hybridization detection, and to monitor DNA damage $[7,11,19,21]$.

Recently EBiS has been used for cancer detection and single cell analysis [22]. Furthermore, impedance changes have been used for biochip detection and to identify the cellular response to an external analyte in real time [23]. To our knowledge, this is the first work that proposes an EBiS biosensor to evaluate the detection of gene expression by its specific hybridization. In this study, we demonstrate that three genes involved in the inflammation process can be measured by means of EBiS on immobilized cDNA. The immobilization of cDNA in a functionalized gold surface has been successfully evaluated by our research group through Atomic Force Microspocpy (AFM) characterization; the surface chemistry was achieved using human DNA as probe with no chemical modification [19].

The results of the present study demonstrate that the bioimpedance of the negative control (gen non-specific) decreases with respect to the other three genes used in this study (NLRP3, IL-1 $\beta$ and Casp-1), in this sense the cDNA immobilization and its hybridization should promote structural changes in the resulting gold surface, which should contribute to an increase in electrical conductivity possibly associated with an apparent increase in the effectiveness of the electric-ion exchange. Such effect could be explained at first by the availability of electrically unstable molecules during the immobilization and hybridization reaction.

Berney's group reported a DNA biosensor that after hybridization induced charged effects, which altered the dielectric properties and was then detected by changes in the measured capacitance [24]. Furthermore, Marquette's group reported an increase in impedance when determining the melting temperature of a 20-mer oligonucleotide [20]. The obtained results of this work are in agreement with Berney's and Marquette's results; an increase in impedance is observed when the hybridization takes place.

It should be noted that unlike Marquette's and Mousavisani's groups that worked with synthetic DNA hybridization, our results show different impedance when hybridizing specific oligonucleotides to identify the expression of genes related to inflammation in a population of obese Mexican children [19]. In a study that could be considered as a previous reference, Ma's group reported a correlation between impedance and DNA fragment length, proposing the use of an electrical system to validate and characterize PCR products [25].
The results obtained in this work show that the integrated relative EBiS values are in agreement with the relative expression of the genes encoding for NLRP3, IL-1 $\beta$, and Casp-1. These findings do not represent a lineal correlation of EBis with the relative gene expression. EBis vs qPCR was done as a simple comparison strategy. In a recent study, a radiofrequency scattering parameter was used as an indirect impedance measurement to detect label-free cDNA. The use of this parameter allowed the detection of different cDNA concentrations. Thus, cDNA at different concentrations promotes changes in the volumetric electrical properties of the analyte [26]. This finding supports the results of this study; bioimpedance spectroscopy changes are observed in genes and negative control assays in relation to the control assay (vehicle), and such changes are associated in general with a modification of the volumetric electrical properties in the electrical-ionic interface.

Electrical Properties of DNA have been studied with controversial conclusions, some authors consider DNA as a molecular wire that can conduct charge carriers, and other conclude its behavior as insulator [27]. In this study we have proposed a simple RC equivalent electrical circuit of a single DNA molecule anchoring on an electrode surface. The model includes resistive and capacitive elements with proposed values to represent the interactions "DNA molecule medium vehicle". The interaction is modeled by $\mathrm{R}_{\mathrm{DNA}}(5 \mathrm{~K} \Omega)$, $C_{\text {DNA }}$ (20uF) in parallel as DNA molecule resistance and capacitance, respectively, and $R_{m}(50 \Omega)$ as the medium resistance.

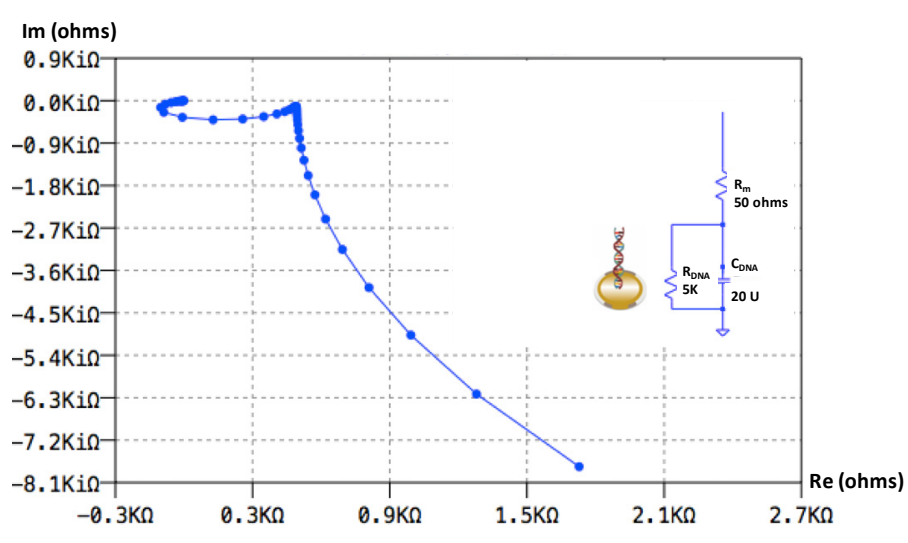

Fig 5. Numerical modelling of a simple DNA molecule anchoring on an electrode surface. Frequency response of the RC equivalent circuit calculated by LTspice IV.

A numerical estimation of the frequency response calculated by LTspice IV showed analogous Nyquiste behaevior mostly consistent with experimental findings (figure 5), thus this basic electrical model proposed might be the key for a best understanding of the biophysical phenomenon.

In conclusion, the combination of a cDNA biosensor that hybridizes with its specific oligonucleotides and the analysis 
of multifrequency electrical bioimpedance changes allow the detection of specific genes.

\section{Conflict of interest}

Authors state no conflict of interest.

\section{Funding statement}

This work was supported by the linstituto Politecnico

Nacional - México [SIP 20171208 and SIP 20181840].

\section{Acknowledgment}

Preliminary results of this work for a single gene detection were presented in the XL National Conference on Biomedical Engineering (CNIB 2017), Monterrey, N.L.-México 2017, and IFMBE Proceedings of the VIII Latin American Conference on Biomedical Engineering (CLAIB 2019), Cancún, Q.R. México 2019. The functionalization techniques for immobilization of cDNA without chemical modifications and biosensor based on multifrequency electrical bioimpedance measurements are supported based on patent applications IPN 2014MX/E/2014/057816 and González CA 2018MX/a/2018/ 009086 - 2017MX/a/2017/010319, respectively.

\section{References}

1. Shafer KJ, Siders WA, Johnson LK, Lukaski HC, Validity of segmental multiple-frequency bioelectrical impedance analysis to estimate body composition of adults across a range of body mass indexes. Nutrition. Vol. 25, no. 1, pp. 25-32, Jan 2009. https://doi.org/10.1016/j.nut.2008.07.004

2. Min-Ho Jun, Soochan Kim, Boncho Ku, JungHee Cho, Kahye Kim, Ho-Ryong Yoo \& Jaeuk U. Kim, Glucose-independent segmental phase angles from multi-frequency bioimpedance analysis to discriminate diabetes mellitus. Scientific Reports. Vol 8, no. 1, pp. 648, Jan 2018. https://doi.org/10.1038/s41598-017-18913-7

3. Bicheng Zhu and Jadranka Travas-Sejdic, PNA versus DNA in electrochemical gene sensing based on conducting polymers: study of charge and surface blocking effects on the sensor signal, Analyst, Vol 143, no. 3, pp. 687-694, Feb 2018. https://doi.org/10.1039/c7an01590a

4. Marsilea Adela Booth, SallyAnn Harbison, Jadranka TravasSejdic, Development of an electrochemical polypyrrole-based DNA sensor and subsequent studies on the effects of probe and target length on performance, Biosens and Bioelectron, Vol. 28, no. 1, pp. 362-367, Oct 2011. https://doi.org/10.1016/j.bios.2011.07.051

5. Ebrahimi M, Bakhsh Raoof J, Ojani R, Design of an electrochemical DNA-based biosensor for selective determination of cadmium ions using a DNA hybridization indicator. International Journal of Biological Macromolecules. Vol. 108, pp 1237-1241, 2018. https://doi.org/10.1016/j.ijbiomac.2017.11.023

6. Wang $\mathrm{H}$, Li H, Huang $\mathrm{Y}$, Xiong $\mathrm{M}$, Wang $\mathrm{F}$, Li C, A label-free electrochemical biosensor for highly sensitive detection of gliotoxina based on DNA nanostructure/MXene nanocomplexes. Biosensors and Bioelectronics. Vol. 142, 111531, 2019. https://doi.org/10.1016/j.bios.2019.111531
7. Zangeneh MM, Norouzi $\mathrm{H}$, Mahmoudi M, Goicoechea HC, Jalalvand $A R$, Fabrication of a novel impedimetric biosensor for label free detection of DNA damage induced by doxorubicin. International Journal of Biological Macromolecules. Vol 124, pp. 963-971, 2019. https://doi.org/10.1016/j.ijbiomac.2018.11.278

8. Tedeschi L, Ciiti L, Domenici C, An integrated approach for the desing and synthesis of oligonucleotide probes and their interferencing to a QCM-basef RNA biosensor. Biosensor and Bioelectronics. Vol. 20, no. 11, pp. 2376-2385, May 2005. https://doi.org/10.1016/j.bios.2004.12.013

9. Teles $\mathrm{F}$ and Fonseca L. Trends in DNA biosensors. Talanta. Vol. 77, no. 2, pp. 606-623, December 2008. https://doi.org/10.1016/j.talanta.2008.07.024

10. Lucarelli F, Tombelli S, Minunni M, Marrazza G, and Mascini M, Electrochemical and piezoelectric DNA biosensors for hybridisation detection. Anal. Chim. Acta. Vol. 609, no. 2, pp. 139-59, Feb 2008. https://doi.org/10.1016/j.aca.2007.12.035

11. Chiorcea-Paquim AM, Enache TA, Oliveira-Brett AM, Electrochemistry of Alzheimer disease amyloid beta peptides. Curr Med Chem. Vol. 8, no. 1, pp. 18, Feb 2018. https://doi.org/10.2174/0929867325666180214112536

12. Kannan S, Begoyan VV, Fedie JR, Xia S, Weseliński ŁJ, Tanasova M, Rao S, Metabolism-Driven High-Throughput Cancer Identification with GLUT5-Specific Molecular Probes. Biosensors (Basel). Vol. 10, no. 2, pp. 39, Apr 2018. https://doi.org/10.3390/bios8020039

13. Turner AP, Biosensors: sense and sensibility. Chem Soc Rev. Vol. 42, no. 8, pp. 3184-3196, Apr 2013.

14. Kuldeep Mahato, Pawan Kumar Maurya, Pranjal Chandra, Fundamentals and commercial aspects of nanobiosensors in point-of-care clinical diagnostics. 3 Biotech. Vol. 8. No. 149, March 2018. https://doi.org/10.1007/s13205-018-1148-8

15. Fan W, Xu Y, Liu Y, Zhang Z, Lu L, Ding Z, Obesity or Overweight, a Chronic Inflammatory Status in Male Reproductive System, Leads to Mice and Human Subfertility. Front Physiol. Vol. 4, no. 8, pp. 1117, Jan 2018. https://doi.org/10.3389/fphys.2017.01117

16. Zhang C, Boini KM, Xia M, Abais JM, Li X, Liu Q, Li PL, Activation of Nod-like receptor protein 3 inflammasomes turns on podocyte injury and glomerular sclerosis in hyperhomocysteinemia. Hypertension. Vol. 60, no. 1, pp. 15462, Jul 2012. https://doi.org/10.1161/hypertensionaha.111.189688

17. Qiu YY, Tang LQ. Roles of the NLRP3 inflammasome in the pathogenesis of diabetic nephropathy. Pharmacol Res. Vol. 114, pp. 251-64, Dec 2016. https://doi.org/10.1016/j.phrs.2016.11.004

18. Ben Ke, Wen Shen, Xiangdong Fang and Qinghua $\mathrm{Wu}$, The NLPR3 inflammasome and obesity-related kidney disease. J Cell Mol Med. Vol. 22, no. 1, pp. 16-24, Jan 2018. https://doi.org/10.1111/jcmm.13333

19. Gómez López M, Pérez-Vielma NM, González Martínez H, Lara Padilla E, Bandala C, González Torres MC, Miliar-García A, NLRP3, IL-1B, and Caspase-1 Gene Transcript Identification and Expression by QCM-D in Obese Children. Journal of Sensors. Vol. 2019, Article ID 4323056, 14 pages, 2019. https://doi.org/10.1155/2019/4323056 
20. C. AMarquette, I Lawrence, C Polychronakos, M. FLawrence, Impedance based DNA chip for direct Tm measurement, Talanta Vol. 56, no. 4, pp. 763-768, March 2002. https://doi.org/10.1016/s0039-9140(01)00633-6

21. Mousavisani S Z, Raoof J B, Ojani R, Bagheryan Z, An impedimetric biosensor for DNA damage detection and study of the protective effect of deferoxamine against DNA damage. Bioelectrochemistry. Vol. 28, pp. 122:142-148, March 2018. https://doi.org/10.1016/j.bioelechem.2018.03.012

22. Valente $V$ and Demosthenous $A$, Wideband FullyProgrammable Dual-Mode CMOS Analogue Front-End for Electrical Impedance Spectroscopy. Sensor (Basel). Vol. 16, no. 8, pp. 1159, Jul 2016. https://doi.org/10.3390/s16081159

23. Fareid Asphahani, Kui Wang, Myo Thein, Omid Veiseh, Sandy Yung, Jian Xu, and Miqin Zhang, Single-cell bioelectrical impedance platform for monitoring cellular response to drug treatment. Phys Biol. Vol. 8, no. 1, 15006, Feb 2012. https://doi.org/10.1088/1478-3975/8/1/015006
24. H. Berney, J. West, E. Haefele, J. Alderman, W. Lane, J.K. Collins, A DNA diagnostic biosensor: development, characterisation and performance. Sensors and Actuators. Vol. 28, pp. 100-108, 2000. https://doi.org/10.1016/s0925-4005(00)00468-8

25. Hanbain MA, Richard W.R., Wallbank, Reza Chaji, Jiahao Li, Yuji Suzuki, Chris Jiggins, Arokia Nathan, An impedance-based integrated biosensor for suspended DNA characterization. Scientific Reports. Vol. 3, pp. 2730, Sep 2013. https://doi.org/10.1038/srep02730

26. Gnaim R, Sheviryov J, Golberg A, Ames G, Oziel M, González CA. Label-free cDNA detection base don radiofrequency scattering parameters: a new approach for an inexpensive gene sensor. Journal of Medical Devices. Vol .14, 014502, March 2020. https://doi.org/10.1115/1.4045909

27. Dekker $C$ and Ratner $M$, Electronic properties of DNA. Physics World. Vol 14, no. 8, pp 29-33, August 2001. 